\title{
Animal regeneration in the era of transcriptomics
}

Loïc Bideau ${ }^{1}$, Pierre Kerner ${ }^{1}$, Jerome Hui ${ }^{2}$, Michel Vervoort ${ }^{1 \#}$, Eve Gazave ${ }^{1 \#}$

1: Université de Paris, CNRS, Institut Jacques Monod, F-75006, Paris, France

2: School of Life Sciences, Simon F.S. Li Marine Science Laboratory, State Key Laboratory of Agrobiotechnology, The Chinese University of Hong Kong, Hong Kong

\#= co-senior and co-corresponding authors

\section{ORCID numbers:}

Pierre Kerner 0000-0003-4001-0812

Jerome Hui 0000-0003-1355-8495

Michel Vervoort 0000-0002-5995-2140

Eve Gazave 0000-0002-4647-6640

\section{Acknowledgements:}

This work was supported by the PROCORE - France/Hong Kong Joint Research Scheme. Work in the Vervoort team is supported by funding from the Labex "Who Am I" laboratory of excellence (No. ANR- 11-LABX-0071) funded by the French Government through its "Investments for the Future" program operated by the Agence Nationale de la Recherche under grant No. ANR-11-IDEX-0005-01, the Centre National de la Recherche Scientifique (CNRS), the INSB department (grant "Diversity of Biological Mechanisms"), the Agence Nationale de la Recherche (ANR grants TELOBLAST no. ANR-16-CE91-0007 and STEM), the "Association pour la Recherche sur le Cancer" (grant PJA 20191209482), and the "Ligue 
Nationale Contre le Cancer" (grant RS20/75-20). JH is funded by the TUYF Charitable Trust. We are grateful to Haley Flom for her diligent proofreading of this manuscript.

\section{Abstract}

Animal regeneration, the ability to restore a lost body part, is a process that has fascinated scientists for centuries. In this review, we first present what regeneration is and how it relates to development, as well as the widespread and diverse nature of regeneration in animals. Despite this diversity, animal regeneration includes three common mechanistic steps: initiation, induction and activation of progenitors, and morphogenesis. In this review article, we summarize and discuss from an evolutionary perspective the recent data obtained for a variety of regeneration models, which have allowed to identify key shared mechanisms that control these main steps of animal regeneration. This review also synthesizes the wealth of high-throughput mRNA sequencing data (bulk mRNA-seq) concerning regeneration which have been obtained in recent years, highlighting the major advances in the regeneration field that these studies have revealed. We stress out that through a comparative approach, these data provide opportunities to further shed light on the evolution of regeneration in animals. Finally, we point out how the use of single-cell mRNA-seq technology and integration with epigenomic approaches may further help researchers to decipher mechanisms controlling regeneration and their evolution in animals. 
Key words: Regeneration, evolution, transcriptomics, bulk RNA-seq, sc RNA-seq, signaling pathways 


\section{What is regeneration?}

\section{- $\quad$ Concept and definition}

From the fantastical features of mythic beasts to the routine tasks of less remarkable organisms, the description of species' restorative properties has only increased our collective fascination for regeneration. Seemingly every civilization has built folklore as well as some basic knowledge on the topic, like the regenerating eye of the ancient Egyptians' god Horus, or the newt-like regenerative feats of the Aztec deity Xolotl, without forgetting the regenerating liver of the ancient Greeks' Prometheus [1]. Regeneration has also drawn the attention of great minds and scientific communities throughout history. Starting from antiquity with Aristotle and Pliny the Elder [1], regeneration studies were especially successful with the works of Abraham Trembley on hydras [2] and Lazzaro Spallanzani's work on salamanders [3], with many important concepts and biological principles discovered in their wake $[4,5]$. However, it seems that no one attempted to sketch a clear definition of regeneration until the seminal work of Thomas Hunt Morgan in 1901 [6]. Morgan juxtaposed three different phenomena that could be covered by the notion of regeneration: egg and embryo regeneration, physiological regeneration, and restorative regeneration. These are better known today as "embryonic regulation" (the compensation of cell or tissue loss during embryogenesis), "homeostatic regeneration" (periodic loss and replacement of cells and tissues such as the gut lining, epidermis, hair, feathers and antlers turn-over) and "restorative regeneration" (replacement of lost body parts or the entire body), respectively $[7,8]$. It is noteworthy that the term "regeneration" is also used for both unicellular organisms and microbial communities, or even ecosystems [9]. We will however restrict our discussion to restorative regeneration processes of individual metazoans in this review. Some authors [10] define this phenomenon as "the restoration, the replacement of a lost body part through traumatic injury (either amputation or autotomy)". It is however not a sufficiently precise definition and may raise potential confusion between healing or scarring and regeneration. It also does not properly clarify the overlap of regeneration and asexual reproduction in some species. Furthermore, one of the main 
disagreements in the domain of regeneration is whether it should be considered as a part of development [8].

\section{- $\quad$ Regeneration versus development}

For many authors, the striking similarities between embryonic development and regeneration make the case for considering the restorative capabilities found in adults as a part of development, which therefore would need to be extended throughout the life of animals. Yet, there are major differences between these processes that would, by contrast, suggest that embryonic development and regeneration are distinct phenomena with an overlapping use of common cellular and genetic mechanisms. There are, for instance, at least eight restorative regeneration-specific processes found in some or all metazoans $[8,11]$

1) Wound healing: the formation of an epithelial barrier against environmental insults necessary for regeneration to take place [12].

2) Immune response: the deployment of the animal immune system at the site of injury seems crucial for normal regeneration in some metazoans [13].

3) Induced reprogramming: resident or distant adult cells - either stem cells or differentiated cells - are activated by the injury and may require unique pathways to recover embryonic-like cell behaviors [11].

4) Transdifferentiation: regeneration depends in some cases on the redifferentiation of dedifferentiated adult cells into different cell types than their original ones [12].

5) System integration: regenerated cells and tissues are integrated and organized in existing differentiated structures, with an emphasis with the continuation of existing systems such as the vascular and nervous systems [11].

6) Nerve dependency: many regeneration events seem to depend on the activity of the established nervous system [14]. 
7) Size recognition and termination: positional identity and positional memory in regenerating structures allow for a harmonious growth until an adequate morphology is reconstructed [15].

8) Proliferation of differentiated cells: only during the regeneration of some organs or structures will fully differentiated cells start dividing again [16].

Despite these specificities, it remains unclear whether these regeneration-specific responses could justify their exclusion from bona fide developmental mechanisms. One way to advance the "regeneration versus development" discussion is to rigorously classify the different types of regeneration living organisms can perform.

\section{- $\quad$ Different types of regeneration}

T.H. Morgan proposed a dualistic grouping of varieties of regeneration based on the requirement of active cell proliferation [6] that to date is still used. At the time regarded as a regeneration expert [17], Morgan compiled a thorough synthesis on earlier regenerative studies, leading him to propose a basic subdivision of regeneration into two general types called epimorphosis and morphallaxis. In epimorphosis, an organism such as a salamander restoring its limb regenerates by recruiting dividing cells, whereas morphallaxis refers to the remodeling of existing tissue, exemplified by a regenerating Hydra.

Further precisions and discussion points followed and researchers also frequently try to discriminate blastemal regeneration (where the process requires the apparition of a mass of undifferentiated cells or blastema) from other types of regeneration. Yet some authors warn that blastemas, while they look alike in many species, may have wildly different origins, ontogenically or evolutionarily [8]. It is also possible to differentiate regeneration processes involving pre-existing stem cells (such as in the regenerative prowess of planarians relying on migrating stem cells called neoblasts), dedifferentiating or transdifferentiating cells, and compensatory regeneration involving dividing differentiated cells (displayed during human liver regeneration for instance). 
Finally, regeneration processes can also be classified depending on the extent of their restorative power. A hierarchical regeneration ladder can therefore be established depending on the structures that can be restored, starting with cell regeneration such as axonal regeneration (which we will exclude from the present review), organ (0) or tissue regeneration such as the restoration of a newt lens, complex structure (CS) regeneration such as that of limbs, and finally whole-body (WB) regeneration such as restoration of a full flatworm from a small body fragment.

All these previous types of regeneration are displayed by at least one member of the multicellular organisms in the tree of life [18], especially in the metazoan lineage [10], which will be our sole point of focus during the remainder of this review.

\section{- $\quad$ Regeneration at the metazoan scale}

Mapping up-to-date data on the regeneration abilities of different metazoan lineages can be a crucial tool to understanding the evolution of regeneration, and discussing whether a single or independent origins of regeneration scenario is more likely $[19,20]$. We therefore generated a tree (Figure 1) encompassing the main metazoan lineages. Some broad observations can be made, such as the lack of WB regeneration capacity in ecdysozoans and vertebrates, or the almost ubiquitous organ/tissue regeneration ability shared by metazoans (with the notable exception of nematodes, and uncertainties regarding tardigrades, onychophorans, and priapulids due to the scarcity of experimental tests). It is noteworthy that this kind of mapping (Figure 1 ) highlights the relatively frequent occurrence of regenerative abilities among metazoans. An experimental bias towards ecdysozoan and vertebrate models may have hindered regenerative research so far, but a recent slew of new and unconventional model systems offers an opportunity to reduce the paucity of our basic knowledge on regeneration [21].

\section{Mechanisms of regeneration}


Despite the high variability of regenerative types and processes displayed by metazoan species, a careful mechanistic analysis revealed what appears to be a conserved, tri-modular organization of the restorative regeneration of complex structures, such as appendages and the whole body [20, 22] (Figure 2). These modules are, by chronological order:

1) Wound closure through the formation of an epithelium and general body response to injury

2) Regeneration induction, usually by the recruitment of progenitors and the formation of a blastema

3) Morphogenesis involving patterning, differentiation, and growth Bound to overlap in some instances, these three successive modules are nevertheless distinct in their nature. A thorough exploration of the processes and genetic pathways involved in these modules in diverse animal models might therefore highlight specificities or conserved similarities at the metazoan level. We will review here the common signals and pathways that have been described as crucial for the transition between wound healing and blastema formation, which constitutes the initiation of regeneration per se. Following that, we will discuss the diversity of both the origins and potency of the progenitors populating the blastema. Lastly, we will highlight the mechanisms that allow the regeneration of functional structures across metazoans.

\section{- Initiation of regeneration in metazoans:}

Three major pathways are emerging as key factors for the initiation of regeneration in various animal species: ROS (reactive oxygen species) signaling, apoptosis, and innate immunity (Figure 2A).

- $\underline{\mathrm{ROS}}$

ROS is an umbrella term used for a wide variety of oxidizers containing oxygen, such as hydrogen peroxide $\left(\mathrm{H}_{2} \mathrm{O}_{2}\right)$ and superoxide anion radical $\left(\mathrm{O}_{2}{ }^{-}\right)$, both of which are produced as metabolic byproducts [23]. They are the cause of oxidative stress and damage, and can lead to cell death; however, they are also involved in cell signaling during various processes, especially regeneration. In vertebrates, notably during zebrafish fin or Xenopus tadpole tail regeneration, ROS are produced very early at the 
wound site and their production is sustained after wound closure [24, 25]. In both species, lowering the level of ROS by inhibiting NADPH oxidase (NOX) activity - which is responsible for their production - strongly reduces the size of the regenerated structure, notably by reducing the number of proliferative cells in the blastema. A similar role has been demonstrated during planarian regeneration, as inhibiting ROS production affects stem cell early differentiation, resulting in an improper regeneration and shortening of the blastema [26]. The importance of ROS has been also acknowledged during Drosophila imaginal disc regeneration, where they activate JNK and p38 signaling pathways which in turn trigger the JAK/STAT pathway required for regeneration [27]. Finally, in the cnidarian Hydra, ROS are produced at the wound site briefly after amputation and might therefore be required for proper regeneration in this species as well [28].

\section{- $\quad$ Cell death}

ROS are known to trigger cell death [29], especially by apoptosis, and there is now clear evidence that apoptosis promotes regeneration through the so-called apoptosis-induced compensatory proliferation (AiP) [30]. During Xenopus tadpole tail regeneration, apoptosis does not follow amputation immediately, but triggers cell proliferation later on [31]. Two rounds of apoptosis happen quite early during zebrafish fin regeneration; the second round co-occurs with the peak of ROS production and triggers subsequent cell proliferation which is crucial for blastema formation [24]. In both species, blocking apoptosis at early time points after amputation impairs regeneration. In planarians, two rounds of apoptosis happen as well, co-occurring with increased cell divisions: the first happens early after amputation and is restricted to the wound region, while the second spreads throughout the organism and happens later during regeneration [32]. While apoptosis is required for tissue remodeling during planarian regeneration [33], a causal link between apoptosis and cell division has not been demonstrated. Lastly, Hydra head regeneration and the sea anemone Nematostella vectensis oral regeneration provide another example of AiP, within cnidarians. In Nematostella, a burst of apoptotic activity appears at the cut site shortly after amputation. Pan-caspase inhibitors hamper both apoptosis and proliferation which subsequently block regeneration [34]. In Hydra, there is an early 
round of apoptosis followed by cell proliferation induced by the diffusion of Wnt molecules from apoptotic bodies [35]. Wnt-containing apoptotic bodies are also found in regenerating zebrafish epidermis and shown to promote proliferation of adjacent stem cells through Wnt signaling [36]. Thus, the chain of events, starting with ROS production which triggers apoptosis and in turn leads to cell proliferation, is emerging as a widely distributed, and possibly conserved, key pathway involved in regeneration initiation in Eumetazoa (Cnidaria plus Bilateria).

- Innate immunity

Innate immunity appears to have an important role during the first steps of regeneration, especially in vertebrates. In regenerating zebrafish fins and salamander limbs, both neutrophils and macrophages populate the wound region quickly after amputation, and the depletion of macrophages shortly after amputation tilts the balance in favor of inflammation, which results in a significant reduction of blastema size and impaired regeneration [37]. Yet, pro-inflammatory cytokines are secreted after amputation as well [13] and were shown to be important for early regeneration: knockdown (KD) of interleukin-8 (IL-8; also known as CXCL8) prevents the recruitment of neutrophils at the wound site and the normal expression of inflammatory genes during the first phases of zebrafish fin regeneration [38]. IL-8 KD delays regeneration and leads to defective recruitment of monocytes and granulocytes during salamander limb regeneration [39]. The implication of innate immunity during regeneration in other phyla is less obvious as the available data are scarcer, but growing evidence hints at a similar importance. In both planarians and Hydra, mRNA-seq data for regenerative tissues show an upregulation of numerous genes annotated as innate immunity-related, notably lectins, metalloproteinases, and putative members of TLR signaling [40, 41].

It is now well-established that ROS signaling, apoptosis, and immune response to injury play major roles in the first steps of regeneration in many animals. ROS production is the earliest signal, being detectable in some species within minutes after the injury, and could serve as a wound sensor. Apoptosis and immune response to injury are shown to start acting slightly later to control activation 
of progenitors and correct patterning of the reformed tissues. It must be noted that those three pathways are extremely intricate and can trigger each other easily, in any direction $[29,42,43]$.

\section{- Recruitment and activation of progenitors:}

We will describe two main aspects of this process, the source or origin of the pool of undifferentiated cells that constitute the blastemal, and the fate and potency of these blastemal cells (Figure 2B).

\section{- Origin of blastemal cells}

In many animals, the blastema is formed by migratory cells that are recruited upon injury. The best and prime instance of such is WB regeneration in planarians. The source of their blastemal cells has triggered a long scientific controversy [44], and it is now fully confirmed that those cells arise from undifferentiated resident stem cells called neoblasts. Indeed, it has been shown in the planarian Schmidtea mediterranea that upon amputation of the anterior-most part of the worm, neoblasts rapidly migrate to the wound and form the blastema [45]. The identification of the distant origin of planarian blastemal cells in turn triggered the search for migratory cells involved in regeneration in other animals. In annelids, such migratory cells were observed in a regeneration context in three species, either through an indirect cell lineage approach by S-phase labelling in Enchytraeus japonensis [46] and Capitella teleta [47], or by using long-term live imaging in Pristina leidyi [48].

Among deuterostomes, regeneration in the colonial ascidians Botrylloides leachi requires the activation of non-circulatory "inner cells" lining the epithelium of blood vessels [49]. Upon injury, those cells detach from the epithelium and migrate towards the wound where they form aggregates [49]. Similarly, in the solitary ascidian Ciona intestinalis, precursors of the regenerating distal oral siphon originate from a proximal organ, the branchial sac, whose cells migrate upon injury towards the wound [50]. There are examples of cell migration in vertebrate's regeneration as well [51]. During zebrafish fin regeneration, the tracking of Di-l-labelled cells proved that migration can occur from distant epithelial and connective tissues towards the blastema in a short time window (up to 2 days after amputation) [52]. In particular, it was shown that, upon amputation, osteoblast progenitor cells 
migrate from their niche in the joints and contribute significantly to the regenerated bones [53]. Migration of regenerative cells also happens in non-bilaterian species. In Hydra, in vivo tracing of Green Fluorescent Protein-producing (GFP+) interstitial stem cells grafted onto wild-type (WT) animals showed that those cells migrate upon amputation towards the wound and replace the lost tissues and stem cells [54]. In Hydractinia echinata, both direct and indirect cell tracking techniques showed that cells forming the head blastema originate from more posterior tissues in the body column [55]. Migration is thus the primary source of regenerative cells in several organisms scattered across eumetazoans, which advocates for the potent ancestrality and conservation of this mechanism in various lineages of regenerative animals. Yet, convergence cannot be excluded.

As such, regeneration does not always rely on migratory cells. During urodele limb regeneration, blastema formation does not depend on distant cells but rather on dedifferentiation of local mature tissues abutting the amputation plane [56]. For instance, it was shown in transgenic lines of adult newts in which myofibers were labelled with fluorescent markers, that myocytes do dedifferentiate during regeneration and proliferate within the blastema [57]. Likewise, grafting experiments showed that nerve cell dedifferentiation contributes to blastema formation in axolotls [58]. During zebrafish fin regeneration, labelling of mature fibroblasts and osteoblasts showed that both cell types dedifferentiate and actively populate the blastema, as well as the newly regenerated tissues [59]. Similarly in mice which have very limited regenerative abilities but are still able to regenerate their digit tip, after amputation axons close to the wound degenerate, which triggers the dedifferentiation of their associated Schwann cells [60]. Those cells participate in the blastema's formation, and are crucial for its proper growth [60]. In the annelid Platynereis dumerilii, indirect evidence by S-phase labelling also suggests that posterior body regeneration mainly occurs through local dedifferentiation [61]. Histological analyses also support the presence of dedifferentiation during regeneration in various species, such as the amphioxus Branchiostoma lanceolatum [62], some echinoderms [63, 64] and the jellyfish Polyorchis penicillatus [65]. Lastly, the blastema can also originate from the activation of local resident stem cells. In Xenopus tadpoles and zebrafish larvae, muscle satellite cells close to the 
wound are activated and populate the blastema [66]. Similarly, satellite cells participate in regeneration for axolotls [57] and larval newts [67]. In zebrafish, both dedifferentiated osteoblasts and undifferentiated cells, the osteoblast progenitors contribute to bone regeneration $[51,53]$. In mice digit tips, most of the blastema is formed thanks to activation of several types of resident stem/progenitor cells [68]. Finally, outside vertebrates, during leg regeneration of the crustacean Parhyale hawaiensis, the blastema is partly formed by normally quiescent cells located in the muscles, resembling vertebrate satellite cells [69].

\section{- Fate and potency of blastemal cells}

A second important aspect is to define the identities of blastemal cells, in particular whether a blastema contains pluripotent and/or tissue-specific (multipotent or unipotent) stem/progenitor cells. As advanced techniques of cell tracking and fate mapping are required for these identities to be properly investigated, data are still scarce. However, two opposite models are emerging. It was hypothesized for a long time that morphologically indistinct blastemal cells constitute a pool of multi/pluripotent progenitors [70]. This assertion was properly proven in the planarian Schmidtea mediterranea in which a subset of neoblasts called 'cNeoblasts' can restore regenerative abilities when injected in small numbers into lethally irradiated hosts [71]. A single cNeoblast can even replenish an entire irradiated animal. Acoels worms (Acoelomorpha) harbor neoblasts as well, even though it is still not clear whether they correspond to a homologous cell type and have the exact same properties [72, 73]. This led to the hypothesis that the involvement of multi/pluripotent stem cells during regeneration could be conserved across bilaterians [74]. As such, in the ascidian Ciona intestinalis, regeneration occurs from a unique stem cell niche, the branchial sac, whose progenitors can give rise to both muscle and nervous tissues [75]. Outside bilaterians, the existence in Hydra of multipotent stem cells, the interstitial cells or i-cells, was well-known for a long time [76] and it has been shown that those stem cells could migrate upon injury and participate in regeneration [54]. Similarly, in Hydractinia, regeneration of the head of a polyp involves the migration of multipotent i-cells [55]. 
In contrast, in vertebrates, amphibians, zebrafish, or mammals, blastemal cells are lineage-restricted stem/progenitor cells. In Xenopus, grafts of GFP+ tissues onto WT animals proved that during tadpole tail regeneration, the spinal cord and notochord are regenerated by their own remnants which dedifferentiate and proliferate at the amputation stump, while muscles are reformed only through the activation of satellite cells [77]. Similar grafting experiments, recently supported by data obtained with CRIPSR/Cas9 technology, led to the same conclusions in axolotls in which cartilage, muscle, or nervous cells are unable to switch to another lineage during regeneration $[58,78]$. The conservation of cell lineages during regeneration is also documented for zebrafish fin regeneration, with a strict separation of fate between osteoblasts, fibroblasts and epidermal cells [79]. Likewise, cell tracing in mice showed a strict restriction of fate during digit tip regeneration between germ layers, and that bone and cartilage on the one hand, and tendons and vasculature on the other hand do not transdifferentiate into one another [68]. Thus, strict lineage restriction during regeneration appears to be a conserved feature among vertebrates. It must however still be noted that there are examples in which mesodermal progenitors, during regeneration, can expand their progeny compared to the one produced in homeostatic conditions $[80,81]$.

\section{- Redifferentiation of the progenitors and morphogenesis}

Another major question is how differentiation of blastemal cells is controlled, especially in terms of size and patterning of regenerated structures, to produce a fully functional structure which usually displays shape, size and function, in accordance with its position in the body (e.g., along anteriorposterior and dorsal-ventral axes), and similar to the one that was amputated. Two main points will be discussed here: how the amputated tissues keep in memory what the structure to be regenerated is, as well as the importance of nervous tissues and reinnervation for proper regeneration (Figure $2 \mathrm{C}$ ).

\section{- Positional memory during regeneration in bilaterians: role of mesodermal tissues}

Planarians, given their outstanding regenerative abilities, are particularly relevant models to study how positional information is encoded, said information being required for the blastema to regenerate the 
proper structure depending on its position along the body of the animal. Witchley and colleagues [82] searched for genes exhibiting a regionalized expression pattern that they refer to as Position Control Genes (PCG). Strikingly, it appears that expression of these genes is almost entirely restricted to the muscles of the body wall. Upon any kind of amputation, their overall pattern is rapidly re-established in the amputated fragments, which allows for the correct specialization of the activated neoblasts forming the blastema [82]. A similar expression of PCG and similar role of muscles have been shown during Hofstenia regeneration [83]. In vertebrates, positional information seems to be encoded in mesodermal tissues as well. In axolotl, grafts of GFP+ tissues into WT animals showed that cartilagederived cells possess proximo-distal identity [58]. Intercalation assays where distal blastemas are grafted upon proximal stumps confirmed that connective tissue cells contain proximo-distal information that allows proper regeneration of the limb [84]. Zebrafish posterior fins are also able to maintain their shape through regeneration with longer peripheral fin rays and shorter central fin rays. Fin ray grafts that are displaced along the proximo-distal axis retain memory of their origin and will not regenerate according to their new position [85]. Given that epidermal tissues of the graft are quickly replaced by the host epidermis, this memory is most likely also carried by the mesoderm [85].

- Importance of innervation

Although proper functional and mechanistic evidence is still scarce in most animals, nerve-dependent regeneration appears to be shared among metazoans (reviewed in [14]). The role of innervation during regeneration was particularly highlighted in the salamander limb in which denervation impedes a blastema to grow and differentiate [86] and rerouting a peripheral nerve underneath an epidermal wound triggers the formation of an ectopic limb [87]. Re-innervation induces a gradient of the growth factor nAG, a ligand for the receptor Prod1 which is crucial for the establishment of the proximo-distal axis during regeneration [88]. Furthermore, it was shown that nerves induce the up-regulation of a histone deacetylase (HDAC) in the blastema just before the differentiation stages. HDAC expression could modify the epigenetic landscape of the blastema and allows its differentiation as the inhibition of HDAC activity results in a severely delayed regeneration [89]. In planarians, nerves were shown to 
be crucial for the patterning of regenerative parts along with cell-cell communication through the gap junctions. Severing the ventral nerve cord and blocking gap junctions leads to the formation of ectopic heads in place of tails [90]. More recently, modeling of planarian regeneration suggested that the diffusion of small molecules alone cannot explain the quick and stereotypical reestablishment of the PCG pattern in body fragments of various sizes [91]. The authors of this study proposed that nerves may provide a scaffold for the directional transport of morphogens, and therefore that initial nerve polarity would determine the antero-posterior axis of regenerating fragments.

As for development, many entangled developmental genes and signaling pathways exert crucial functions in the later stages of regeneration, i.e. the patterning and morphogenesis of the regenerated structures. In the next section, we review how genome-wide transcriptomic analyses allowed to identify such genes and pathways involved in regeneration in diverse animals and how these approaches may help to better understand the evolution of regeneration in animals.

\section{Insights from regeneration bulk transcriptomic data}

While having been intensively studied morphologically during the first part of the $20^{\text {th }}$ century, animal regeneration withdrew from developmental biology's center stage with the rise of genetic and molecular developmental studies in the 70s [10, 92]. Indeed, the main developmental biology models that emerged at that time (i.e. Drosophila, C. elegans, Mus musculus), while having been instrumental in providing insights into many biological questions, were of limited interest to study restorative regeneration due to their restricted regenerative abilities [10]. Conversely, the majority of key regeneration model species were not easily amenable for functional analysis [93]. We are, however, currently witnessing a strong revival of interest from the scientific community for regeneration [92, 93]. New mechanistic studies of regeneration are mainly fostered by technical advances, notably highthroughput sequencing technologies that are currently widely and successfully applied to less conventional regeneration models. 
- Bulk mRNA-seq data uncover the transcriptional profiles of various types of regeneration in many non-model species throughout metazoans

High-throughput mRNA sequencing, commonly called bulk mRNA-seq, is a popular technology used nowadays to monitor changes in gene expression that accompany complex and dynamic biological processes [94]. This unbiased approach provides a timed in-depth overall view of the transcriptomic landscape of cells participating in regeneration. By estimating and comparing relative gene expression levels with a high degree of accuracy, identifying thousands of differentially-expressed genes that may play crucial roles during regeneration steps is now a fairly straightforward process. In the last ten years, a huge amount of mRNA-seq data was gathered, with more than 100 studies on species (around 50) belonging to almost all metazoan lineages with regenerative abilities (Figures 1 and 3, Supplementary Table 1). Unsurprisingly, the main bilaterian model systems for regeneration, namely flatworms (Platyhelminthes [95]), salamanders and Xenopus (Lissamphibia [96]), and the zebrafish (Actinopterygii [97]) encompass the majority of bulk mRNA-seq studies (Figure 3, Supp. Table 1). In addition, many studies investigated regeneration in Mammalia, although their regenerative potential is more limited, driven by possible applications for regenerative medicine [98]. In contrast, while non-bilaterian lineages, namely Porifera [99], Placozoa, Ctenophora, and Cnidaria harbor extensive regeneration capabilities [10], very few studies have investigated their transcriptomic changes during regeneration, with the noticeable exceptions of Cnidaria [100, 101]. In terms of phylogenetic distribution, information from important regenerative groups, i.e., Ctenophora [102], Nemertea [103], Cephalochordata [62], and Mollusca [104] (as well as more under-looked ones: Brachiopoda, Phoronida, Ectoprocta, Gastotricha, Entoprocta, Chaetognatha, Rotifera, Priapulida, Xenoturbellida [10]) are still missing in current existing datasets.

For the other main clades (Acoelomorpha, Annelida, Arthropoda, Hemichordata, Tunicata, Mammalia), data come mostly from one or two species, which prevents any generalization to the entire group. A single species cannot be considered as representative of its whole lineage, especially concerning a 
character as labile as regeneration. Regeneration has a rather rich evolutionary history, as speciesspecific innovations are frequent within closely-related species often exhibiting very different regenerative abilities $[12,103,105]$. In contrast, in Platyhelminthes, up to seven species displaying various regeneration features have been molecularly studied, uncovering the regulatory network at the origin of head regeneration (see below) [106-111]. So far, our knowledge of changes in transcription during regeneration concerns more than 50 species and various types of regeneration, including whole-body regeneration of Cnidaria, Acoelomorpha, Tunicata, and Planaria, regeneration of complex structures such as body axis (Annelida, Hemichordata), appendages (Actinopterygii, Lissamphibia and Arthropoda), and organ regeneration (Vertebrata) (Figure 1, Supp. Table1).

The overwhelming majority of these studies has been performed on adult animals, with only a couple of studies investigating larval stages (in sea stars [112]), juvenile (Xenopus tadpoles [113]) and newborn (rats [114] and mice [115]), which leaves a gap in our greater comprehension of regeneration processes. The majority of available data are series of mRNA-seq data at different time points after an amputation or a wound, which cover the three main aforementioned steps of regeneration and therefore allow us to finely highlight the dynamics of the process. In a couple of earlier studies [116, 117], the data provided were restricted to one specific regenerative stage (usually a blastema stage) or time point, and are consequently much less informative. Altogether, these bulk mRNA-seq studies made significant strides toward our understanding of regeneration and have given us the opportunity to further shed light on its evolution using a comparative approach $[98,112,118]$.

\section{- Major advances in the regeneration field revealed by bulk mRNA-seq}

New questions on regeneration can be addressed thanks to bulk mRNA-seq data and their comparative analyses. Four of those major topics will be discussed below.

\section{- Regeneration versus development}

Whether adult injury-induced regeneration is a post-embryonic developmental process or a phenomenon distinct from development is a long-standing, unresolved scientific question. On the one 
hand, regeneration has its proper evolutionary history and contains specific features not found during development [8]. On the other hand, restoring complex tissue structures upon injury involves the reactivation of developmental processes that are specific to the regenerated structure and that have to remain available in adult stages [119]. Transcriptomic data allowed us to address this question by defining, in a couple of species, to which extent regeneration and developmental programs share molecular commonalities and present specificities. A recent study on the cnidarian Nematostella vectensis (sea anemone) sought to answer this question for the first time on a greater scale, thanks to the comparison of a large time series of mRNA-seq data for development and regeneration [34, 101]. This study revealed that regeneration uses a partial and rewired embryonic gene regulatory network (GRN), with regeneration-specific modules driving cellular events unique to regeneration, such as apoptosis [34]. Along the same line, another recent study investigated the process of skeletogenesis in the echinoderm Amphiura filiformis (brittle star) during both embryogenesis and arm regeneration [120]. This analysis focused mainly on the role of the FGF signaling pathway during skeletogenesis and supports the hypothesis that regeneration recapitulates development in this species. While these seminal analyses pointed out similarities between development and regeneration in both the investigated species, more studies are required to fully address the question of the relationships between development and regeneration in Metazoa.

\section{- Patterning}

Like an embryo, a regenerating body region must be meticulously patterned to ensure that only the appropriate structures are formed, at the proper location, and with the correct size and shape [7]. Patterning during regeneration is regulated by positional memory, a cellular property that involves adult cells spared from injury and maintains necessary information for structures' replacement [121]. Positional memory has been studied mainly in the context of appendages and main body axis regeneration. Comparison of tissue transcriptomes at different anatomical locations was performed during appendage regeneration in both zebrafish [121] and axolotls [122]. This enabled the identification of several candidate transcripts involved in positional memory along the proximo-distal 
axis of the limb (such as the RNA-binding protein cirbp, which plays a cytoprotective role, and kazald1, whose $\mathrm{KO}$ in blastema impairs regeneration) and caudal fin (such as transcription factors of the dlx and msx families, known to be involved in the patterning of developing appendages), paving the way for future mechanistic studies [121, 122]. Which programs drive anterior/posterior (AP) regeneration in bilaterians, but also apical/basal (or oral/aboral) regeneration in cnidarians, is a prominent question in the field. In Hydra, while a similar initial transcriptional response is observed early after amputation on either side of the oral/aboral axis, dramatically different programs are set up later on, involving BMP signaling on the basal part and Wnt- $\beta$-catenin pathway on the apical side [123]. The important role of the latter during apical regeneration has been described in both Hydra and Hydractinia in which knockdown (KD) of Wnt3 impedes regeneration upon decapitation [35, 124]. Furthermore, incubation of Wnt3 protein induces the formation of ectopic "heads" on body columns [125]. Similarly, in Nematostella, Wnt- $\beta$-catenin members are highly expressed in the oral pole compared to the aboral one [126]. Strikingly, the Wnt- $\beta$-catenin pathway is also a central regulator of axial polarity during regeneration of a variety of bilaterian species. In the larva of the sea star Patiria miniata, Wnt ligands and Frizzled receptors are up-regulated posteriorly during regeneration, suggesting that Wnt- $\beta$-catenin may have a role in AP patterning [112]. In the annelid Aeolosoma viride, inhibition or overactivation of the Wnt- $\beta$-catenin pathway impairs head regeneration [127]. In the planarian Schmidtea, Wnt- $\beta$ catenin activation specifies posterior regeneration while its inhibition (through notum) specifies anterior regeneration $[107,128]$. KD of $\beta$-catenin results in the regeneration of a head in place of a tail [128], and KD of notum in the opposite outcome [129]. Similarly, in the Acoelomorpha Hofstenia, Wnt$\beta$-catenin is activated during posterior regeneration [130, 131]: KD of Wnt- $\beta$-catenin positive mediators results in anterior-like structures in place of the tail, and KD of Wnt inhibitors in tail structures in place of the head [73]. A gene regulatory network for posterior regeneration initiation has recently been described, with a Wnt ligand (Wnt-3) being transcriptionally regulated by an early wound response factor (Egr) [131]. The Wnt- $\beta$-catenin pathway is also responsible for other crucial functions in later stages of regeneration. In axolotls, its inhibition prevents normal regeneration and 
results in the formation of a spiky outgrowth [132], while its global activation results in malformations of regenerated skeletal tissues [133]. Similarly, in zebrafish fin regeneration, inhibition of Wnt- $\beta$ catenin signaling early after amputation prevents blastema formation [132], while later on during blastema differentiation, its inhibition hinders proper bone calcification [134]. During mouse digit tip regeneration, Wnt- $\beta$-catenin pathway activation in the epidermal progenitors is crucial for the differentiation of the regenerated nail, but also for blastema growth and overall regeneration [135]. Besides highlighting the role of the Wnt- $\beta$-catenin in regeneration success, the comparison of head and tail regeneration transcriptomes in Schmidtea led to another important and unanticipated observation. While their transcriptome profiles were initially very different, they later converged to a shared core regenerative program [107]. In stunning contrast, in the Syllidae Sphaerosyllis hystrix and Syllis gracilis (annelid worms), anterior and posterior regenerations do not rely on a common transcriptomic landscape: the posterior regeneration program is indeed more related to the one used during posterior growth (growth by addition of segments in the posterior body part of uninjured juvenile animals) than to the head regeneration program [136].

\section{- Variations in regenerative capabilities}

Regenerative capacity varies greatly across animals but also across the life cycle of a given species [10]. Why do certain animals possess substantial regenerative capacities (at least during a specific period of their life cycle), while others lack such amazing abilities? Answering this crucial question is fundamental to implementing medical strategies aiming to unlock the regenerative potential of humans. To start answering this question, researchers have exploited the vast richness of regeneration patterns in animals. Xenopus is an amphibian species that does not display life-long regenerative capacity, as this ability is restricted to the pre-metamorphic larval stages (with the exception of the retina) [137]. LeeLiu and colleagues investigated Xenopus spinal cord regeneration during its regenerative and nonregenerative stages, revealing differences in the timing of the transcriptional response and in the inventory of regulated transcripts involved in broad biological processes including neurogenesis, metabolism, immune response and inflammation, cell cycle, development, and response to stress 
[113]. The axolotl Ambystoma mexicanum is another amphibian which can regenerate its appendages in a nerve-dependent manner: regeneration does not occur in denervated limbs [96]. Interestingly, repeated limb amputations lead to regeneration defects and failure [138]. Two studies explored the transcriptomic landscape of those impaired regenerations in comparison to normal regenerating limbs, laying down a detailed blueprint of mis-regulated genes hindering regeneration, such as amphiregulin, an EGF-like ligand [138, 139]. Similarly, comparison between regenerating tails versus nonregenerating limbs were performed in the lizard Podarcis muralis, revealing the importance of small nucleolar RNAs and Wnt signaling pathways for tail regeneration [140]. In planarians, while some species such as Schmidtea are able to reform a full animal from a single pluripotent stem cell [71], many others lack (or have limited) regenerative abilities and notably are unable to regenerate their anterior part upon amputation [106, 110]. Procotyla fluviatilis and Dendrocoelum lacteum, for example, fail to regenerate their head if amputated too posteriorly along their body axis. Comparative transcriptomic analysis between regeneration-competent and non-competent tissues revealed that the Wnt signaling pathway is aberrantly activated in non-competent tissues and that downregulation of this pathway is sufficient to restore head regeneration from regeneration-non-competent tissues $[106,110]$. These two seminal papers revealed that manipulating a single signaling pathway can be sufficient to reverse the evolutionary loss of regenerative potential in planarians.

\section{- Evolutionary history of regeneration}

The origin and evolution of regeneration in animals is a long-standing debate, and while several ecological reasons and evolutionary hypotheses have been proposed, why and how regeneration abilities evolved remain a mystery [10]. Nevertheless, as regeneration is found in species that belong to all of the main branches of the metazoan tree, the possibility that this ability is an ancestral feature of animals and might therefore rely on homologous mechanisms and genetic networks is a tempting hypothesis that requires careful examination [21]. A broad comparative approach, using the tremendously increasing quantity of mRNA-seq data obtained in the recent years, for a large spectrum of metazoan species, will be a compelling strategy to address this question. A couple of attempts have 
already been made, leading to a patchy inventory of similar, homologous, or co-opted components of regeneration networks in distantly-related species $[112,118,126]$. Hence, comparisons between Hydra, Schmidtea, and Patiria whole-body regeneration revealed the common involvement, during the immediate-early phase of regeneration, of cell death/apoptosis-related genes, MAPK signalingassociated genes (such as Jnk), and the transcription factor-encoding gene Egr. At later stages of regeneration, the Wnt signaling pathway is mandatory to specify the axis in those three species before the occurrence of a massive cell proliferation event which is associated with the expression of different genes. Another computational and broad comparison between Hydra, Schmidtea and the echinoderm Apostichopus japonicus (sea cucumber) identified 18 common differentially-expressed genes with functions related to metabolic processes and signaling pathways such as Wnt and cadherin [118]. Our own analysis of the extensive bibliography related to bulk mRNA-seq data during regeneration (Supp. Table 1) also points out several major signaling pathway components, in addition to the Wnt- $\beta$-catenin pathway already mentioned, notably Jak/STAT [113, 141, 142], Notch [126, 143-145], and MAPK [108, $142,143,146]$, in particular downstream of FGF/FGFR signaling $[120,123,147,148]$, which are dynamically expressed in various regeneration contexts and steps (Figure 4). This analysis also unveils the importance of less-studied regulators, such as those linked to epigenetic modifications $[112,143$, 149], non-coding RNAs [146, 147], and importantly many unknown novel or species-specific regeneration genes $[107,113,122,141,147,150]$.

In summary, we outlined in this section that a comparative evolutionary study of regeneration is a powerful strategy to tackle key regeneration-linked questions. There is clearly much more to learn from the wealth of information gathered so far from mRNA-seq analyses, and this will undoubtedly be pursued in the next coming years.

\section{Perspectives}

While bulk transcriptomics has duly demonstrated its usefulness to identify genes and pathways involved in complex phenomena such as development or regeneration, it is not applicable for the 
identification and characterization of cellular states nor for the comprehension of how those states change over the course of such processes [151]. Bulk analysis eliminates crucial information by averaging signals from individual cells and does not allow to discriminate between changes due to gene regulation and those due to modifications in cell type composition. In recent years, NGS-based technologies for transcriptomics have been exploring a new direction for characterizing individual cells, i.e., single-cell RNA sequencing (scRNA-seq) (e.g., $[151,152])$. Methods have been developed to use scRNA-seq to characterize cell populations and track distinct cell lineages during embryonic development (e.g., $[153,154])$. These approaches have also been used to understand regeneration processes in different species across the animal kingdom, including WBR in the planarian Schmidtea [155-160], anterior body regeneration in the earthworm Eisenia [161], tail regeneration in Xenopus [162, 163], leg regeneration in the axolotl Ambystoma [164-166], fin and heart regeneration in zebrafish [167-169], and digit regeneration in mice [170-173].

These studies revealed the diversity of cell populations during regeneration and unveiled previously unrecognized cell types in the blastema, such as multipotent mesenchymal-like progenitors producing various connective tissue lineages during axolotl limb regeneration $[164,165]$, regeneration-organizing cells which belong to the wound epidermis and act as a signaling center during Xenopus tail regeneration [162], or rare and transient cell types required for WBR in Schmidtea [160]. Another very promising path to further unravel regeneration mechanisms is to combine mRNA-seq (both bulk and scRNA-seq) with epigenomic approaches. In a recently-published seminal study, bulk mRNA-seq, scRNA-seq, and chromatin immunoprecipitation sequencing (ChIP-seq) were used to compare regeneration in two distantly-related teleosts, zebrafish and african killifish Nothobranchius. This study revealed an evolutionarily-conserved regeneration response program involving specific regenerationresponsive enhancers, some of which may have been repurposed for other functions in vertebrates with poor regeneration abilities such as mammals [168]. Application of these transcriptomic and epigenomic approaches, and more generally of multi-omics approaches [174] to an increasing number 
of regeneration models will undoubtedly help decipher fundamental regeneration mechanisms and their evolution in animals. 


\section{Figure Legends:}

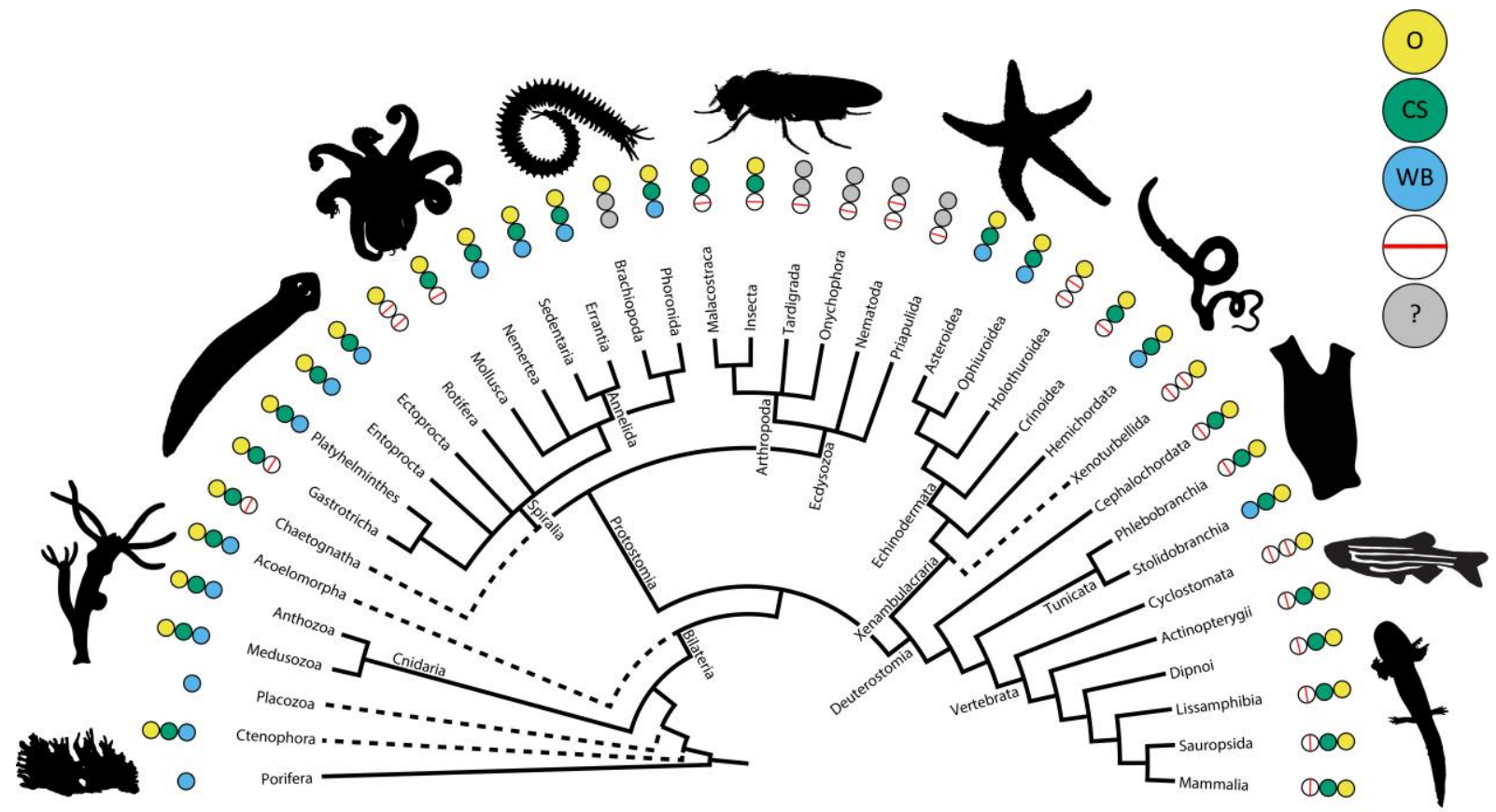

Figure 1: Mapping regeneration types on the metazoan phylogenetic tree. Most metazoan phyla include representative species with documented abilities to regenerate organs ( $\mathrm{O}$, yellow circles) and complex structures (CS, green circles). While absent (struck-through circles) in all vertebrates, ecdysozoans, and some other scattered lineages, whole-body regeneration (WB, blue circles) is a widespread phenomenon among Metazoa. Lack of substantiated data (gray circles) is mostly observed in Brachiopoda and Ecdysozoa. Phylogenetic tree topology is derived from recent metazoans' phylogenies $[175,176]$, with still-controversial lineage positioning depicted with dotted lines. Porifera, Hydra, flatworm, octopus, Platynereis, Drosophila, sea-star, enteropneust, Ciona, zebrafish, and axolotl silhouettes are adapted from PhyloPic.org (not to scale) [Illustrators: Hillewaert Keesey, Duygu Özpolat, Michelle Site, Jake Warner, Yan Wong]. 


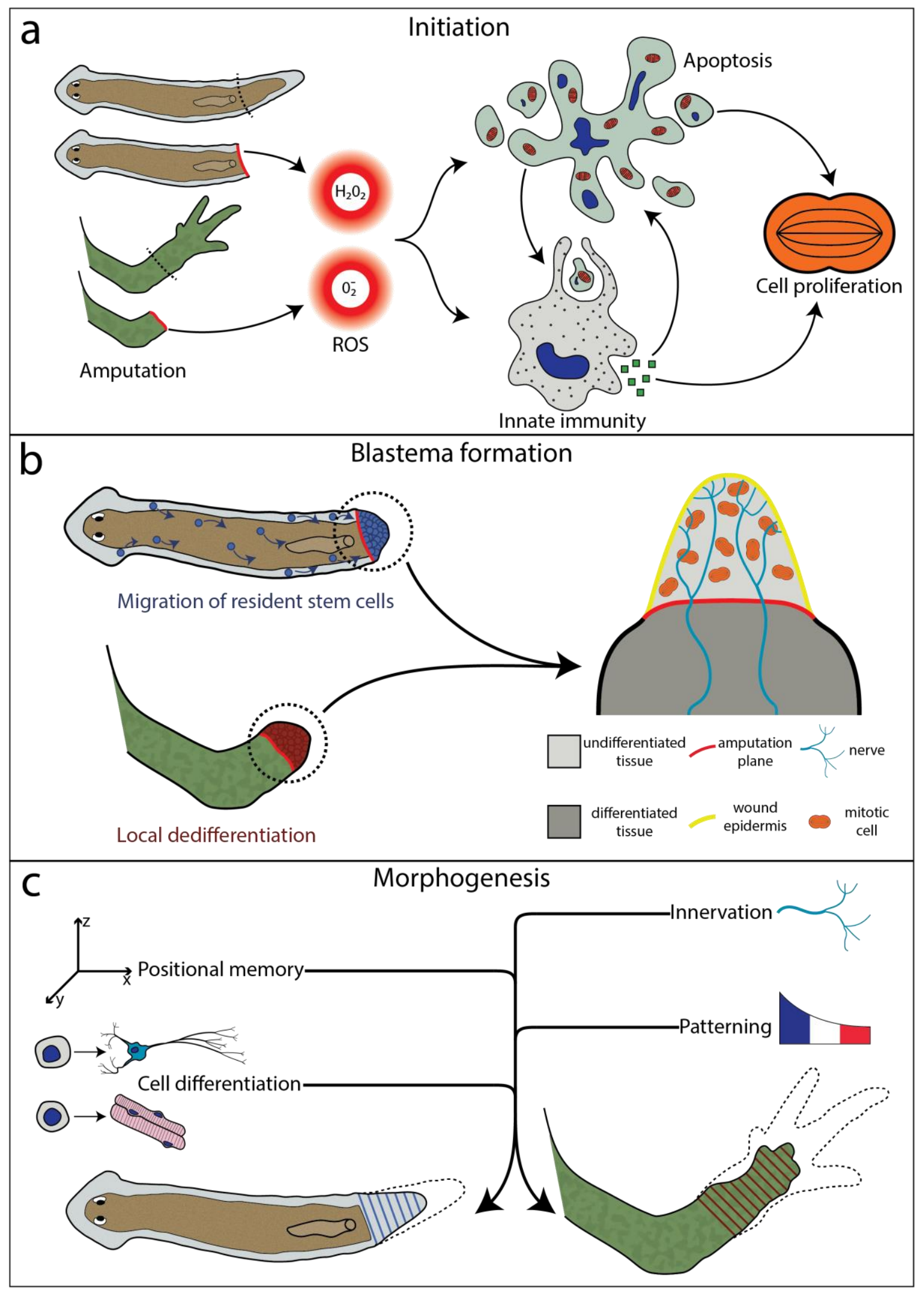

Figure 2: Key common steps in eumetazoan regeneration. A: Initiation of regeneration. Within minutes after the amputation, reactive oxygen species (ROS) are produced at the wound site. Their 
accumulation promotes apoptosis, and activates innate immunity. Innate immunity is also activated by apoptotic cells and produces pro-inflammatory cytokines (green squares) that sustain apoptosis. Surrounding cells enter mitosis through apoptosis-induced proliferation (AiP) and the diffusion of mitogenic pro-inflammatory cytokines (green squares). B: Blastema formation. A blastema, whatever is its origin (migration of resident stem cells or local dedifferentiation) is always composed of undifferentiated mesenchymal cells covered by a wound epidermis. Cell proliferation within the blastema as well as its innervation are crucial for a successful regeneration. C: Morphogenesis. Four important aspects of this complex step are depicted: role of innervation, positional memory of the remaining tissue, patterning of the regenerated region in particular in term of axes, and cell differentiation. More details can be found in the main text.

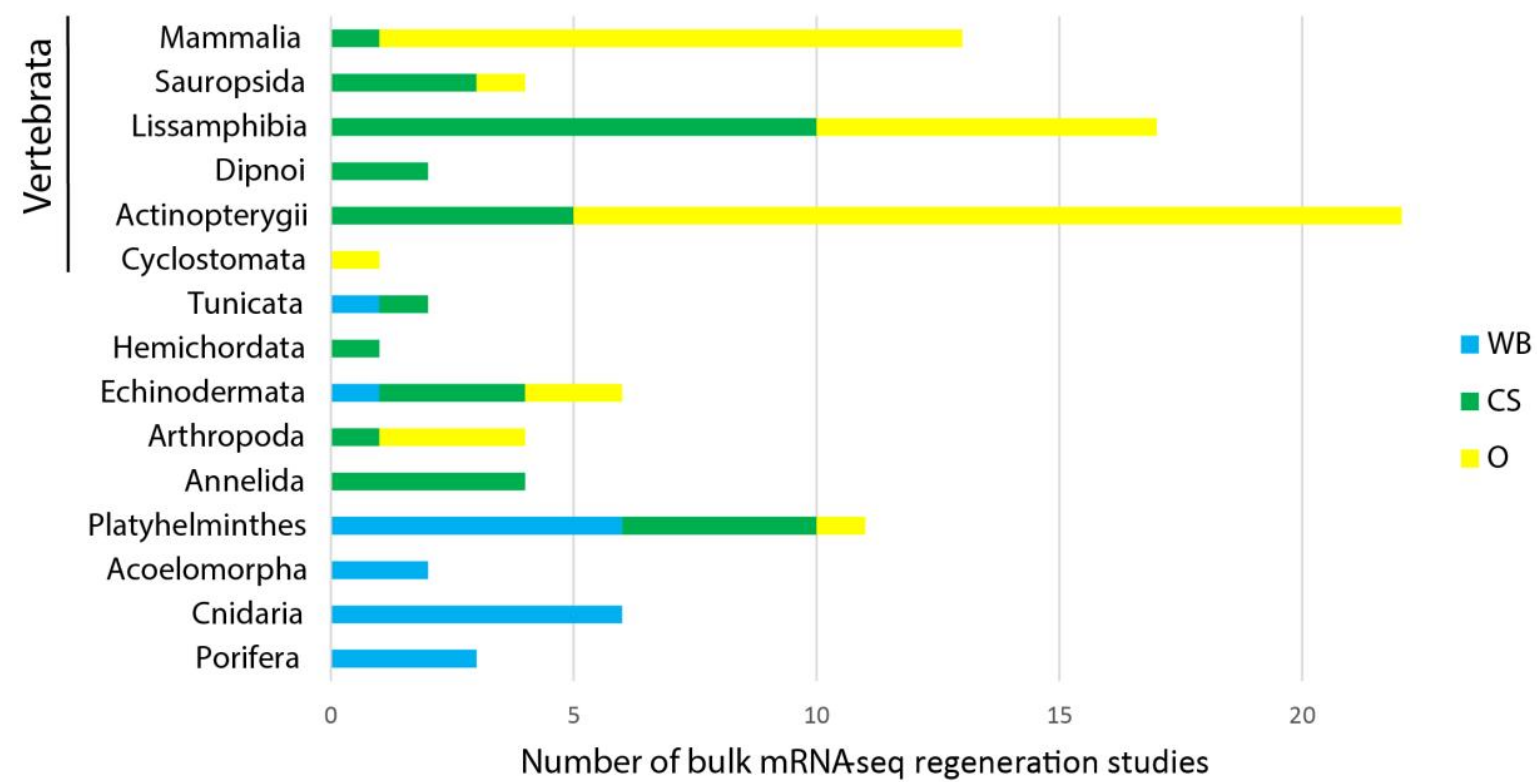

Figure 3: Diversity and number of bulk mRNA-seq regeneration studies. Major groups of metazoans and number of regeneration studies dealing with whole-body regeneration (WB), complex structure regeneration (CS) and organ regeneration (O) in each group are mentioned. 


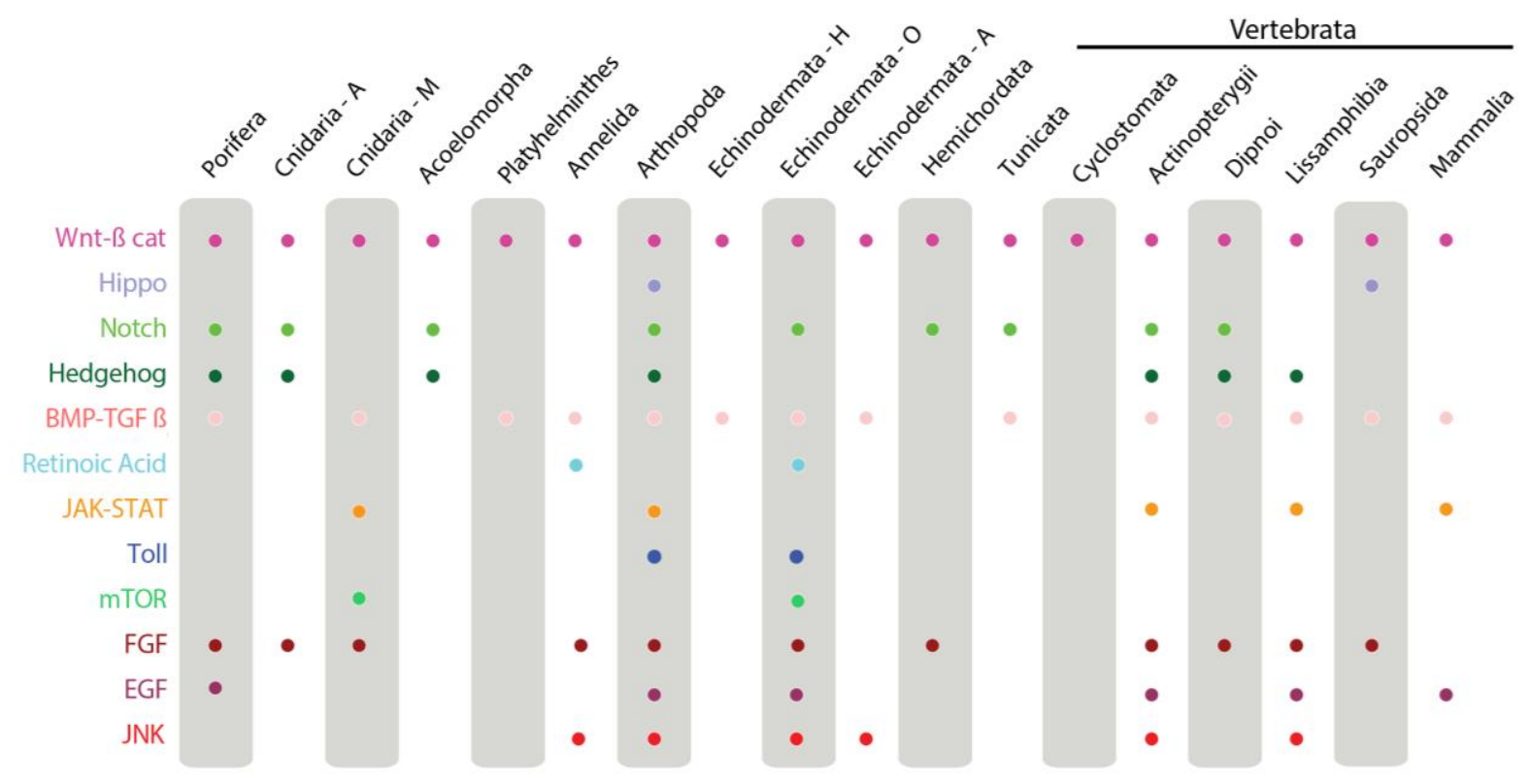

Figure 4: Majors signaling pathways' components are dynamically expressed in various metazoan

regeneration contexts. Transcriptomic data highlight the potential importance of 13 major signaling pathways during regeneration of 15 main metazoan lineages. Circles indicate that at least one transcriptomic study reports differential expression of those pathway components. Cnidaria $-A=$ Anthozoa; Cnidaria $-\mathrm{M}=$ Medusozoa; Echinodermata $-\mathrm{H}=$ Holothuroidea; Echinodermata $-\mathrm{O}=$ Ophiuroidea; Echinodermata $-\mathrm{A}=$ Asteroidea .

\section{Bibliography}

1. Elliott, S.A. and A.S. Alvarado, Planarians and the History of Animal Regeneration: Paradigm Shifts and Key Concepts in Biology. Methods Mol Biol, 2018. 1774: p. 207-239.

2. Trembley, A., et al., Mémoires pour servir à l'histoire d'un genre de polypes d'eau douce, à bras en forme de cornes. 1744: chez Jean \& Herman Verbeek.

3. Spallanzani, L., Prodromo di un'opera da imprimersi sopra le riproduzioni animali dato in luce da Spallanzani. 1768: Montanari.

4. Dinsmore, C.E., A History of Regeneration Research: Milestones in the Evolution of a Science. 1991: Cambridge University Press.

5. Keller, J., Die ungeschlechtliche Fortpflanzung der Süsswasserturbellarien. Jen Zeit Naturw, 1894. 94: p. 3823-3827.

6. Morgan, T.H., Regeneration. 1901, New York: Macmillan. 
7. Poss, K.D., Advances in understanding tissue regenerative capacity and mechanisms in animals. Nat Rev Genet, 2010. 11(10): p. 710-22.

8. Vervoort, M., Regeneration and Development in Animals. Biological Theory, 2011. 6(1): p. 2535.

9. MacCord, K. and J. Maienschein, Understanding regeneration at different scales. Elife, 2019. 8.

10. Bely, A.E. and K.G. Nyberg, Evolution of animal regeneration: re-emergence of a field. Trends Ecol Evol, 2010. 25(3): p. 161-70.

11. Barresi, M.J.F. and S.F. Gilbert, Developmental Biology. 2020: Oxford University Press, Incorporated.

12. Brockes, J.P. and A. Kumar, Comparative aspects of animal regeneration. Annu Rev Cell Dev Biol, 2008. 24: p. 525-49.

13. Godwin, J.W., A.R. Pinto, and N.A. Rosenthal, Macrophages are required for adult salamander limb regeneration. Proc Natl Acad Sci U S A, 2013. 110(23): p. 9415-20.

14. Sinigaglia, C. and M. Averof, The multifaceted role of nerves in animal regeneration. Curr Opin Genet Dev, 2019. 57: p. 98-105.

15. Uemoto, T., G. Abe, and K. Tamura, Regrowth of zebrafish caudal fin regeneration is determined by the amputated length. Sci Rep, 2020. 10(1): p. 649.

16. Sanchez Alvarado, A. and P.A. Tsonis, Bridging the regeneration gap: genetic insights from diverse animal models. Nat Rev Genet, 2006. 7(11): p. 873-84.

17. Sunderland, M.E., Regeneration: Thomas Hunt Morgan's window into development. J Hist Biol, 2010. 43(2): p. 325-61.

18. Coffman, J.A., Regenerative Potential Across Species: An Eco-Evo-Devo Perspective, in Epigenetics and Regeneration, D. Palacios, Editor. 2019, Academic Press. p. 197-214.

19. Slack, J.M., Animal regeneration: ancestral character or evolutionary novelty? EMBO Rep, 2017. 18(9): p. 1497-1508.

20. Tiozzo, S. and R.R. Copley, Reconsidering regeneration in metazoans: an evo-devo approach. Frontiers in Ecology and Evolution, 2015. 3(67).

21. Lai, A.G. and A.A. Aboobaker, EvoRegen in animals: Time to uncover deep conservation or convergence of adult stem cell evolution and regenerative processes. Dev Biol, 2018. 433(2): p. 118-131.

22. Galliot, B. and S. Chera, The Hydra model: disclosing an apoptosis-driven generator of Wntbased regeneration. Trends Cell Biol, 2010. 20(9): p. 514-23.

23. Sies, H. and D.P. Jones, Reactive oxygen species (ROS) as pleiotropic physiological signalling agents. Nat Rev Mol Cell Biol, 2020. 21(7): p. 363-383.

24. Gauron, C., et al., Sustained production of ROS triggers compensatory proliferation and is required for regeneration to proceed. Sci Rep, 2013. 3: p. 2084.

25. Love, N.R., et al., Amputation-induced reactive oxygen species are required for successful Xenopus tadpole tail regeneration. Nat Cell Biol, 2013. 15(2): p. 222-8.

26. Pirotte, N., et al., Reactive Oxygen Species in Planarian Regeneration: An Upstream Necessity for Correct Patterning and Brain Formation, in Oxidative Medicine and Cellular Longevity. 2015.

27. Santabarbara-Ruiz, P., et al., ROS-Induced JNK and p38 Signaling Is Required for Unpaired Cytokine Activation during Drosophila Regeneration. PLoS Genet, 2015. 11(10): p. e1005595.

28. Vriz, S., S. Reiter, and B. Galliot, Cell death: a program to regenerate. Curr Top Dev Biol, 2014. 108: p. 121-51.

29. Mittal, M., et al., Reactive oxygen species in inflammation and tissue injury. Antioxid Redox Signal, 2014. 20(7): p. 1126-67.

30. Fan, Y. and A. Bergmann, Apoptosis-induced compensatory proliferation. The Cell is dead. Long live the Cell! Trends Cell Biol, 2008. 18(10): p. 467-73.

31. Tseng, A.S., et al., Apoptosis is required during early stages of tail regeneration in Xenopus laevis. Dev Biol, 2007. 301(1): p. 62-9.

32. Pellettieri, J., et al., Cell death and tissue remodeling in planarian regeneration. Dev Biol, 2010. 338(1): p. 76-85. 
33. Beane, W.S., et al., Bioelectric signaling regulates head and organ size during planarian regeneration. Development, 2013. 140(2): p. 313-22.

34. Warner, J.F., et al., Regeneration is a partial redeployment of the embryonic gene network. BioRxiv, 2019.

35. Chera, S., et al., Apoptotic cells provide an unexpected source of Wnt3 signaling to drive hydra head regeneration. Dev Cell, 2009. 17(2): p. 279-89.

36. Brock, C.K., et al., Stem cell proliferation is induced by apoptotic bodies from dying cells during epithelial tissue maintenance. Nat Commun, 2019. 10(1): p. 1044.

37. Petrie, T.A., et al., Macrophages modulate adult zebrafish tail fin regeneration. Development, 2014. 141(13): p. 2581-91.

38. de Oliveira, S., et al., CXCl8 (IL-8) mediates neutrophil recruitment and behavior in the zebrafish inflammatory response. J Immunol, 2013. 190(8): p. 4349-59.

39. Tsai, S.L., C. Baselga-Garriga, and D.A. Melton, Blastemal progenitors modulate immune signaling during early limb regeneration. Development, 2019. 146(1).

40. Peiris, T.H., K.K. Hoyer, and N.J. Oviedo, Innate immune system and tissue regeneration in planarians: an area ripe for exploration. Semin Immunol, 2014. 26(4): p. 295-302.

41. Wenger, Y., et al., Injury-induced immune responses in Hydra. Semin Immunol, 2014. 26(4): p. 277-94.

42. Yang, Y., et al., Programmed cell death and its role in inflammation. Mil Med Res, 2015. 2: p. 12.

43. Fogarty, C.E. and A. Bergmann, Killers creating new life: caspases drive apoptosis-induced proliferation in tissue repair and disease. Cell Death Differ, 2017. 24(8): p. 1390-1400.

44. Baguna, J., The planarian neoblast: the rambling history of its origin and some current black boxes. Int J Dev Biol, 2012. 56(1-3): p. 19-37.

45. Wenemoser, D. and P.W. Reddien, Planarian regeneration involves distinct stem cell responses to wounds and tissue absence. Dev Biol, 2010. 344(2): p. 979-91.

46. Sugio, M., et al., Stem cells in asexual reproduction of Enchytraeus japonensis (Oligochaeta, Annelid): Proliferation and migration of neoblasts. Development, Growth \& Differentiation, 2012. 54(4): p. 439-450.

47. de Jong, D.M. and E.C. Seaver, Investigation into the cellular origins of posterior regeneration in the annelid Capitella teleta. Regeneration (Oxf), 2018. 5(1): p. 61-77.

48. Zattara, E.E., K.W. Turlington, and A.E. Bely, Long-term time-lapse live imaging reveals extensive cell migration during annelid regeneration. BMC Dev Biol, 2016. 16: p. 6.

49. Rinkevich, Y., et al., Piwi positive cells that line the vasculature epithelium, underlie whole body regeneration in a basal chordate. Dev Biol, 2010. 345(1): p. 94-104.

50. Jeffery, W.R., Distal Regeneration Involves the Age Dependent Activity of Branchial Sac Stem Cells in the Ascidian Ciona intestinalis. Regeneration (Oxf), 2015. 2(1): p. 1-18.

51. Sehring, I.M. and G. Weidinger, Recent advancements in understanding fin regeneration in zebrafish. Wiley Interdiscip Rev Dev Biol, 2020. 9(1): p. e367.

52. Poleo, G., et al., Cell proliferation and movement during early fin regeneration in zebrafish. Dev Dyn, 2001. 221(4): p. 380-90.

53. Ando, K., et al., Osteoblast Production by Reserved Progenitor Cells in Zebrafish Bone Regeneration and Maintenance. Dev Cell, 2017. 43(5): p. 643-650 e3.

54. Boehm, A.M. and T.C. Bosch, Migration of multipotent interstitial stem cells in Hydra. Zoology (Jena), 2012. 115(5): p. 275-82.

55. Bradshaw, B., K. Thompson, and U. Frank, Distinct mechanisms underlie oral vs aboral regeneration in the cnidarian Hydractinia echinata. Elife, 2015. 4: p. e05506.

56. Stocum, D.L. and J.A. Cameron, Looking proximally and distally: 100 years of limb regeneration and beyond. Dev Dyn, 2011. 240(5): p. 943-68.

57. Sandoval-Guzman, T., et al., Fundamental differences in dedifferentiation and stem cell recruitment during skeletal muscle regeneration in two salamander species. Cell Stem Cell, 2014. 14(2): p. 174-87. 
58. Kragl, M., et al., Cells keep a memory of their tissue origin during axolotl limb regeneration. Nature, 2009. 460(7251): p. 60-5.

59. Stewart, S. and K. Stankunas, Limited dedifferentiation provides replacement tissue during zebrafish fin regeneration. Dev Biol, 2012. 365(2): p. 339-49.

60. Johnston, A.P., et al., Dedifferentiated Schwann Cell Precursors Secreting Paracrine Factors Are Required for Regeneration of the Mammalian Digit Tip. Cell Stem Cell, 2016. 19(4): p. 433-448.

61. Planques, A., et al., Morphological, cellular and molecular characterization of posterior regeneration in the marine annelid Platynereis dumerilii. Dev Biol, 2019. 445(2): p. 189-210.

62. Somorjai, I.M., et al., Vertebrate-like regeneration in the invertebrate chordate amphioxus. Proc Natl Acad Sci U S A, 2012. 109(2): p. 517-22.

63. Fan, T., et al., Patterns and cellular mechanisms of arm regeneration in adult starfish Asterias rollestoni bell. Journal of Ocean University of China, 2011. 10(3): p. 255-262.

64. Di Benedetto, C., et al., Echinoderm regeneration: an in vitro approach using the crinoid Antedon mediterranea. Cell Tissue Res, 2014. 358(1): p. 189-201.

65. Lin, Y.C., N.G. Grigoriev, and A.N. Spencer, Wound healing in jellyfish striated muscle involves rapid switching between two modes of cell motility and a change in the source of regulatory calcium. Dev Biol, 2000. 225(1): p. 87-100.

66. Rodrigues, A.M., et al., Skeletal muscle regeneration in Xenopus tadpoles and zebrafish larvae. BMC Dev Biol, 2012. 12(1): p. 9.

67. Tanaka, E.M., The Molecular and Cellular Choreography of Appendage Regeneration. Cell, 2016. 165(7): p. 1598-1608.

68. Rinkevich, Y., et al., Germ-layer and lineage-restricted stem/progenitors regenerate the mouse digit tip. Nature, 2011. 476(7361): p. 409-13.

69. Konstantinides, N. and M. Averof, A common cellular basis for muscle regeneration in arthropods and vertebrates. Science, 2014. 343(6172): p. 788-91.

70. Stocum, D.L., The urodele limb regeneration blastema. Determination and organization of the morphogenetic field. Differentiation, 1984. 27(1): p. 13-28.

71. Wagner, D.E., I.E. Wang, and P.W. Reddien, Clonogenic neoblasts are pluripotent adult stem cells that underlie planarian regeneration. Science, 2011. 332(6031): p. 811-6.

72. De Mulder, K., et al., Characterization of the stem cell system of the acoel Isodiametra pulchra. BMC Developmental Biology, 2009. 9(1): p. 69.

73. Srivastava, M., et al., Whole-body acoel regeneration is controlled by Wht and Bmp-Admp signaling. Curr Biol, 2014. 24(10): p. 1107-13.

74. Gehrke, A.R. and M. Srivastava, Neoblasts and the evolution of whole-body regeneration. Curr Opin Genet Dev, 2016. 40: p. 131-137.

75. Jeffery, W.R., Progenitor targeting by adult stem cells in Ciona homeostasis, injury, and regeneration. Dev Biol, 2019. 448(2): p. 279-290.

76. David, C.N., Interstitial stem cells in Hydra: multipotency and decision-making. Int J Dev Biol, 2012. 56(6-8): p. 489-97.

77. Gargioli, C. and J.M. Slack, Cell lineage tracing during Xenopus tail regeneration. Development, 2004. 131(11): p. 2669-79.

78. Flowers, G.P., L.D. Sanor, and C.M. Crews, Lineage tracing of genome-edited alleles reveals high fidelity axolotl limb regeneration. Elife, 2017. 6: p. e25726.

79. Tu, S. and S.L. Johnson, Fate restriction in the growing and regenerating zebrafish fin. Dev Cell, 2011. 20(5): p. 725-32.

80. Morrison, J.I., P. Borg, and A. Simon, Plasticity and recovery of skeletal muscle satellite cells during limb regeneration. FASEB J, 2010. 24(3): p. 750-6.

81. Tornini, V.A., et al., Live fate-mapping of joint-associated fibroblasts visualizes expansion of cell contributions during zebrafish fin regeneration. Development, 2017. 144(16): p. 2889-2895.

82. Witchley, J.N., et al., Muscle cells provide instructions for planarian regeneration. Cell Rep, 2013. 4(4): p. 633-41. 
83. Raz, A.A., et al., Acoel regeneration mechanisms indicate an ancient role for muscle in regenerative patterning. Nat Commun, 2017. 8(1): p. 1260.

84. Nacu, E., et al., Connective tissue cells, but not muscle cells, are involved in establishing the proximo-distal outcome of limb regeneration in the axolotl. Development, 2013. 140(3): p. 5138.

85. Shibata, E., et al., Robust and local positional information within a fin ray directs fin length during zebrafish regeneration. Dev Growth Differ, 2018. 60(6): p. 354-364.

86. Brockes, J.P., Mitogenic growth factors and nerve dependence of limb regeneration. Science, 1984. 225(4668): p. 1280-7.

87. Endo, T., et al., The accessory limb model: an alternative experimental system of limb regeneration. Methods Mol Biol, 2015. 1290: p. 101-13.

88. Kumar, A., et al., Molecular basis for the nerve dependence of limb regeneration in an adult vertebrate. Science, 2007. 318(5851): p. 772-7.

89. Wang, M.H., et al., Nerve-mediated expression of histone deacetylases regulates limb regeneration in axolotls. Dev Biol, 2019. 449(2): p. 122-131.

90. Oviedo, N.J., et al., Long-range neural and gap junction protein-mediated cues control polarity during planarian regeneration. Dev Biol, 2010. 339(1): p. 188-99.

91. Pietak, A., et al., Neural control of body-plan axis in regenerating planaria. PLoS Comput Biol, 2019. 15(4): p. e1006904.

92. Gazave, E. and E. Rottinger, 7th Euro Evo Devo meeting: Report on the "Evolution of regeneration in Metazoa" symposium. J Exp Zool B Mol Dev Evol, 2019.

93. Grillo, M., N. Konstantinides, and M. Averof, Old questions, new models: unraveling complex organ regeneration with new experimental approaches. Curr Opin Genet Dev, 2016. 40: p. 2331.

94. Lowe, R., et al., Transcriptomics technologies. PLoS Comput Biol, 2017. 13(5): p. e1005457.

95. Ivankovic, M., et al., Model systems for regeneration: planarians. Development, 2019. 146(17).

96. Joven, A., A. Elewa, and A. Simon, Model systems for regeneration: salamanders. Development, 2019. 146(14).

97. Marques, I.J., E. Lupi, and N. Mercader, Model systems for regeneration: zebrafish. Development, 2019. 146(18).

98. lismaa, S.E., et al., Comparative regenerative mechanisms across different mammalian tissues. NPJ Regen Med, 2018. 3: p. 6.

99. Kenny, N.J., et al., Towards the identification of ancestrally shared regenerative mechanisms across the Metazoa: A Transcriptomic case study in the Demosponge Halisarca caerulea. Mar Genomics, 2018. 37: p. 135-147.

100. Vogg, M.C., B. Galliot, and C.D. Tsiairis, Model systems for regeneration: Hydra. Development, 2019. 146(21).

101. Warner, J.F., et al., NVERTX: a gene expression database to compare embryogenesis and regeneration in the sea anemone Nematostella vectensis. Development, 2018. 145(10).

102. Ramon-Mateu, J., et al., Regeneration in the ctenophore Mnemiopsis leidyi occurs in the absence of a blastema, requires cell division, and is temporally separable from wound healing. BMC Biol, 2019. 17(1): p. 80.

103. Zattara, E.E., et al., A phylum-wide survey reveals multiple independent gains of head regeneration in Nemertea. Proc Biol Sci, 2019. 286(1898): p. 20182524.

104. Imperadore, P., et al., Nerve degeneration and regeneration in the cephalopod mollusc Octopus vulgaris: the case of the pallial nerve. Sci Rep, 2017. 7: p. 46564.

105. Bely, A.E., Evolutionary loss of animal regeneration: pattern and process. Integr Comp Biol, 2010. 50(4): p. 515-27.

106. Sikes, J.M. and P.A. Newmark, Restoration of anterior regeneration in a planarian with limited regenerative ability. Nature, 2013. 500(7460): p. 77-80. 
107. Kao, D., D. Felix, and A. Aboobaker, The planarian regeneration transcriptome reveals a shared but temporally shifted regulatory program between opposing head and tail scenarios. BMC Genomics, 2013. 14: p. 797.

108. Qin, Y.F., et al., Transcriptome profiling and digital gene expression by deep-sequencing in normal/regenerative tissues of planarian Dugesia japonica. Genomics, 2011. 97(6): p. 364-71.

109. Almazan, E.M.P., et al., Girardia dorotocephala transcriptome sequence, assembly, and validation through characterization of piwi homologs and stem cell progeny markers. Dev Biol, 2018. 433(2): p. 433-447.

110. Liu, S.Y., et al., Reactivating head regrowth in a regeneration-deficient planarian species. Nature, 2013. 500(7460): p. 81-4.

111. Wasik, K., et al., Genome and transcriptome of the regeneration-competent flatworm, Macrostomum lignano. Proc Natl Acad Sci U S A, 2015. 112(40): p. 12462-7.

112. Cary, G.A., et al., Analysis of sea star larval regeneration reveals conserved processes of wholebody regeneration across the metazoa. BMC Biol, 2019. 17(1): p. 16.

113. Lee-Liu, D., et al., Genome-wide expression profile of the response to spinal cord injury in Xenopus laevis reveals extensive differences between regenerative and non-regenerative stages. Neural Dev, 2014. 9: p. 12.

114. Pibiri, M., et al., Global gene expression profile of normal and regenerating liver in young and old mice. Age (Dordr), 2015. 37(3): p. 9796.

115. Wang, Z., et al., Mechanistic basis of neonatal heart regeneration revealed by transcriptome and histone modification profiling. Proc Natl Acad Sci U S A, 2019. 116(37): p. 18455-18465.

116. Blythe, M.J., et al., A dual platform approach to transcript discovery for the planarian Schmidtea mediterranea to establish RNAseq for stem cell and regeneration biology. PLoS One, 2010. 5(12): p. e15617.

117. Sun, L., et al., Large scale gene expression profiling during intestine and body wall regeneration in the sea cucumber Apostichopus japonicus. Comparative Biochemistry and Physiology DGenomics \& Proteomics, 2011. 6(2): p. 195-205.

118. Fumagalli, M.R., S. Zapperi, and C.A.M. La Porta, Regeneration in distantly related species: common strategies and pathways. NPJ Syst Biol Appl, 2018. 4: p. 5.

119. Benenati, G., J.I. Montoya-Burgos, and B. Galliot, Towards a parsimonious analysis of regeneration and self-repair in animal evolution, in Proceedings of the Seventh International Workshop on Information Processing in Cells and Tissues (IPCAT 2007), N.C.T.o. Scheper, Editor. 2007, Jesus College Oxford: Oxford, United Kingdom. p. 90-100.

120. Czarkwiani, A., et al., FGF signalling plays similar roles in development and regeneration of the skeleton in the brittle star Amphiura filiformis. BioRxiv, 2019.

121. Rabinowitz, J.S., et al., Transcriptomic, proteomic, and metabolomic landscape of positional memory in the caudal fin of zebrafish. Proc Natl Acad Sci U S A, 2017. 114(5): p. E717-E726.

122. Bryant, D.M., et al., A Tissue-Mapped Axolotl De Novo Transcriptome Enables Identification of Limb Regeneration Factors. Cell Rep, 2017. 18(3): p. 762-776.

123. Wenger, Y., et al., Generic and context-dependent gene modulations during Hydra whole body regeneration. BioRxiv, 2019.

124. Duffy, D.J., et al., Wnt signaling promotes oral but suppresses aboral structures in Hydractinia metamorphosis and regeneration. Development, 2010. 137(18): p. 3057-66.

125. Lengfeld, T., et al., Multiple Wnts are involved in Hydra organizer formation and regeneration. Dev Biol, 2009. 330(1): p. 186-99.

126. Schaffer, A.A., et al., A transcriptional time-course analysis of oral vs. aboral whole-body regeneration in the Sea anemone Nematostella vectensis. BMC Genomics, 2016. 17: p. 718.

127. Chen, C.-Y., W.-T. Yueh, and J.-H. Chen, Canonical Wnt Signaling is Involved in Anterior Regeneration of the Annelid Aeolosoma viride. bioRxiv, 2020: p. 2020.03.01.972448.

128. Petersen, C.P. and P.W. Reddien, A wound-induced Wnt expression program controls planarian regeneration polarity. Proc Natl Acad Sci U S A, 2009. 106(40): p. 17061-6. 
129. Petersen, C.P. and P.W. Reddien, Polarized notum activation at wounds inhibits Wnt function to promote planarian head regeneration. Science, 2011. 332(6031): p. 852-5.

130. Gehrke, A.R., et al., Acoel genome reveals the regulatory landscape of whole-body regeneration. Science, 2019. 363(6432).

131. Ramirez, A.N., K. Loubet-Senear, and M. Srivastava, A Regulatory Program for Initiation of Wnt Signaling during Posterior Regeneration. Cell Rep, 2020. 32(9): p. 108098.

132. Kawakami, Y., et al., Wnt/beta-catenin signaling regulates vertebrate limb regeneration. Genes Dev, 2006. 20(23): p. 3232-7.

133. Wischin, S., et al., Chemical activation of Wnt/beta-catenin signalling inhibits innervation and causes skeletal tissue malformations during axolotl limb regeneration. Mech Dev, 2017. 144(Pt B): p. 182-190.

134. Wehner, D., et al., Wnt/beta-catenin signaling defines organizing centers that orchestrate growth and differentiation of the regenerating zebrafish caudal fin. Cell Rep, 2014. 6(3): p. 46781.

135. Takeo, M., et al., Wnt activation in nail epithelium couples nail growth to digit regeneration. Nature, 2013. 499(7457): p. 228-32.

136. Ribeiro, R.P., et al., Comparative transcriptomics in Syllidae (Annelida) indicates that posterior regeneration and regular growth are comparable, while anterior regeneration is a distinct process. Bmc Genomics, 2019. 20(1).

137. Phipps, L.S., et al., Model systems for regeneration: Xenopus. Development, 2020. 147(6).

138. Bryant, D.M., et al., Identification of regenerative roadblocks via repeat deployment of limb regeneration in axolotls. NPJ Regen Med, 2017. 2: p. 30.

139. Wu, C.H., et al., De novo transcriptome sequencing of axolotl blastema for identification of differentially expressed genes during limb regeneration. BMC Genomics, 2013. 14: p. 434.

140. Vitulo, N., et al., Transcriptome analysis of the regenerating tail vs. the scarring limb in lizard reveals pathways leading to successful vs. unsuccessful organ regeneration in amniotes. Dev Dyn, 2017. 246(2): p. 116-134.

141. Petersen, H.O., et al., A Comprehensive Transcriptomic and Proteomic Analysis of Hydra Head Regeneration. Mol Biol Evol, 2015. 32(8): p. 1928-47.

142. Vizcaya-Molina, E., et al., Damage-responsive elements in Drosophila regeneration. Genome Res, 2018. 28(12): p. 1852-1866.

143. Mashanov, V.S., O.R. Zueva, and J.E. Garcia-Arraras, Transcriptomic changes during regeneration of the central nervous system in an echinoderm. BMC Genomics, 2014. 15: p. 357.

144. Zondag, L.E., et al., Uncovering the pathways underlying whole body regeneration in a chordate model, Botrylloides leachi using de novo transcriptome analysis. BMC Genomics, 2016. 17: p. 114.

145. Luttrell, S.M., et al., Head regeneration in hemichordates is not a strict recapitulation of development. Dev Dyn, 2016. 245(12): p. 1159-1175.

146. $\mathrm{Xu}, \mathrm{C}$., et al., Transcriptional analysis of scar-free wound healing during early stages of tail regeneration in the green anole lizard, Anolis carolinensis. Journal of Immunology and Regenerative Medicine, 2020. 7.

147. Bhambri, A., et al., Large scale changes in the transcriptome of Eisenia fetida during regeneration. PLoS One, 2018. 13(9): p. e0204234.

148. Hutchins, E.D., et al., Transcriptomic analysis of tail regeneration in the lizard Anolis carolinensis reveals activation of conserved vertebrate developmental and repair mechanisms. PLoS One, 2014. 9(8): p. e105004.

149. Bando, T., et al., Analysis of RNA-Seq data reveals involvement of JAK/STAT signalling during leg regeneration in the cricket Gryllus bimaculatus. Development, 2013. 140(5): p. 959-64.

150. Arenas Gómez, C.M., et al., Using transcriptomics to enable a plethodontid salamander (Bolitoglossa ramosi) for limb regeneration research. BMC Genomics, 2018. 19(1): p. 704.

151. Trapnell, C., Defining cell types and states with single-cell genomics. Genome Res, 2015. 25(10): p. 1491-8. 
152. Wang, Y. and N.E. Navin, Advances and applications of single-cell sequencing technologies. Mol Cell, 2015. 58(4): p. 598-609.

153. Cao, J., et al., The single-cell transcriptional landscape of mammalian organogenesis. Nature, 2019. 566(7745): p. 496-502.

154. Pijuan-Sala, B., et al., A single-cell molecular map of mouse gastrulation and early organogenesis. Nature, 2019. 566(7745): p. 490-495.

155. Scimone, M.L., et al., Two FGFRL-Wnt circuits organize the planarian anteroposterior axis. Elife, 2016. 5.

156. Molinaro, A.M. and B.J. Pearson, In silico lineage tracing through single cell transcriptomics identifies a neural stem cell population in planarians. Genome Biol, 2016. 17: p. 87.

157. Wurtzel, O., I.M. Oderberg, and P.W. Reddien, Planarian Epidermal Stem Cells Respond to Positional Cues to Promote Cell-Type Diversity. Dev Cell, 2017. 40(5): p. 491-504 e5.

158. Plass, M., et al., Cell type atlas and lineage tree of a whole complex animal by single-cell transcriptomics. Science, 2018. 360(6391).

159. Fincher, C.T., et al., Cell type transcriptome atlas for the planarian Schmidtea mediterranea. Science, 2018. 360(6391).

160. Benham-Pyle, B.W., et al., Identification of rare transient somatic cell states induced by injury and required for whole-body regeneration. BioRxiv, 2020.

161. Shao, Y., et al., Genome and single-cell RNA-sequencing of the earthworm Eisenia andrei identifies cellular mechanisms underlying regeneration. Nat Commun, 2020. 11(1): p. 2656.

162. Aztekin, C., et al., Identification of a regeneration-organizing cell in the Xenopus tail. Science, 2019. 364(6441): p. 653-658.

163. Kakebeen, A.D., et al., Chromatin accessibility dynamics and single cell RNA-Seq reveal new regulators of regeneration in neural progenitors. Elife, 2020. 9.

164. Gerber, T., et al., Single-cell analysis uncovers convergence of cell identities during axolotl limb regeneration. Science, 2018. 362(6413).

165. Leigh, N.D., et al., Transcriptomic landscape of the blastema niche in regenerating adult axolotl limbs at single-cell resolution. Nature Communications, 2018. 9(1): p. 5153.

166. Rodgers, A.K., J.J. Smith, and S.R. Voss, Identification of immune and non-immune cells in regenerating axolotl limbs by single-cell sequencing. Exp Cell Res, 2020. 394(2): p. 112149.

167. Cao, J., et al., Single epicardial cell transcriptome sequencing identifies Caveolin 1 as an essential factor in zebrafish heart regeneration. Development, 2016. 143(2): p. 232-43.

168. Wang, W., et al., Changes in regeneration-responsive enhancers shape regenerative capacities in vertebrates. Science, 2020. 369(6508).

169. Hou, Y., et al., Cellular diversity of the regenerating caudal fin. Sci Adv, 2020. 6(33): p. eaba2084.

170. Storer, M.A., et al., Acquisition of a Unique Mesenchymal Precursor-like Blastema State Underlies Successful Adult Mammalian Digit Tip Regeneration. Developmental Cell, 2020. 52(4): p. 509-524.e9.

171. Vaughan, A.E., et al., Lineage-negative progenitors mobilize to regenerate lung epithelium after major injury. Nature, 2015. 517(7536): p. 621-5.

172. Carr, M.J., et al., Mesenchymal Precursor Cells in Adult Nerves Contribute to Mammalian Tissue Repair and Regeneration. Cell Stem Cell, 2019. 24(2): p. 240-256 e9.

173. Johnson, G.L., E.J. Masias, and J.A. Lehoczky, Cellular Heterogeneity and Lineage Restriction during Mouse Digit Tip Regeneration at Single-Cell Resolution. Dev Cell, 2020. 52(4): p. 525540 e5.

174. Hasin, Y., M. Seldin, and A. Lusis, Multi-omics approaches to disease. Genome Biol, 2017. 18(1): p. 83.

175. Feuda, R., et al., Improved Modeling of Compositional Heterogeneity Supports Sponges as Sister to All Other Animals. Current Biology, 2017. 27(24): p. 3864-+.

176. Laumer, C.E., et al., Revisiting metazoan phylogeny with genomic sampling of all phyla. Proc Biol Sci, 2019. 286(1906): p. 20190831. 
